\title{
Cuidado pré-natal e integralidade: revisão de escopo
}

\author{
Prenatal care and comprehensiveness: scoping review \\ Atención prenatal y integralidad del cuidado: revisión del alcance
}

Recebido: 15/09/2021 | Revisado: 22/09/2021 | Aceito: 24/09/2021 | Publicado: 25/09/2021

\author{
Melina Renata Blascke Barbieri \\ ORCID: https://orcid.org/0000-0001-7295-1268 \\ Universidade Federal de São Carlos, Brasil \\ E-mail:mblascke@yahoo.com.br \\ Nayara Girardi Baraldi \\ ORCID: https://orcid.org/0000-0003-0124-8174 \\ Universidade de São Paulo, Brasil \\ E-mail: nayyzinha@usp.br \\ Laís Fumincelli \\ ORCID: https://orcid.org/0000-0001-9895-8214 \\ Universidade Federal de São Carlos, Brasil \\ E-mail: laisfumincelli@ufscar.br \\ Bruna Felisberto de Souza \\ ORCID: https://orcid.org/0000-0003-1858-8896 \\ Universidade Federal de São Carlos, Brasil \\ E-mail: brunaf.sc@hotmail.com \\ Monika Wernet \\ ORCID: https://orcid.org/0000-0002-1194-3261 \\ Universidade Federal de São Carlos, Brasil \\ E-mail: monika.wernet@gmail.com \\ Márcia Regina Cangiani Fabbro \\ ORCID: https://orcid.org/0000-0003-2601-8818 \\ Universidade Federal de São Carlos, Brasil \\ E-mail: mfabbbro@gmail.com
}

\begin{abstract}
Resumo
Objetivo: mapear e refletir o estado da arte da integralidade no cuidado pré-natal na atenção primária. Metodologia: scoping review, conduzida conforme recomendações do Instituto Joanna Briggs. Foram consultadas as bases de dados/bibliotecas digitais nacionais e internacionais, no período de 1980-2019. Os estudos foram selecionados e analisados segundo a propositura da integralidade do cuidado proposta por Ayres, a saber, eixo das necessidades, eixo das articulações, eixo das interações e eixo das finalidades. Resultados: foram incluídos e analisados, 26 estudos; a maioria publicado nos anos 2000, sendo 17 estudos nacionais e 9 internacionais. O alcance da integralidade no cuidado pré-natal esteve quase que exclusivamente articulada ao eixo das necessidades. $\mathrm{O}$ eixo das articulações e as demais mostram dificuldades no alcance dos saberes e práticas interdisciplinares, apontando necessidade de mudanças na postura profissional, articulação de redes de atenção à saúde, cooperação entre setores da saúde e sociais, participação e mobilização social. Conclusão: o estado da arte revelou que o cuidado pré-natal, sobretudo edificado pelas relações que pouco se movem para o alcance da integralidade, acaba por dificultar a construção deste princípio no cotidiano das práticas.
\end{abstract}

Palavras-chave: Atenção primária à saúde; Cuidado pré-natal; Integralidade em saúde; Revisão.

\begin{abstract}
Objective: to map and discuss the state of the art of comprehensiveness in prenatal care in primary care. Methodology: review of scope guided by the research protocol of the Joanna Briggs Institute. Publications in national and international digital databases/libraries, dated between 1980-2019 were consulted. The studies were selected and analyzed according to the proposal of comprehensive care proposed by Ayres, namely the axis of needs, axis of articulations, axis of interactions and axis of purposes. Results: 26 studies were included and analyzed; the most published in the 2000s, with 17 national and 9 international studies. The achievement of comprehensiveness in prenatal care was almost exclusively linked to the axis of needs. The articulation axis and the other show difficulties in reaching interdisciplinary knowledge and practices, pointing to the need for changes in professional attitude, articulation of health care networks, cooperation between health and social sectors, participation and social mobilization. Conclusion: the state of the art revealed that prenatal care, especially built by relationships that move little towards achieving comprehensiveness, ends up hindering the construction of this principle in everyday practices. Keywords: Primary health care; Prenatal care; Integrality in health; Review.
\end{abstract}




\begin{abstract}
Resumen
Objetivo: mapear y reflejar el estado del arte de la integralidad en la atención prenatal en atención primaria. Metodología: revisión del alcance, realizada según lo recomendado por el Instituto Joanna Briggs. Se consultaron bases de datos / bibliotecas digitales nacionales e internacionales en el período 1980-2019. Los estudios fueron seleccionados y analizados de acuerdo con la propuesta de atención integral de Ayres, es decir, eje de necesidades, eje de articulaciones, eje de interacciones y eje de propósitos. Resultados: se incluyeron y analizaron 26 estudios; la mayoría se publicó en la década de 2000, con 17 estudios nacionales y 9 internacionales. El logro de la integralidad en la atención prenatal estuvo casi exclusivamente ligado al eje de necesidades. El eje de articulación y los demás muestran dificultades para alcanzar conocimientos y prácticas interdisciplinares, apuntando a la necesidad de cambios en la actitud profesional, articulación de redes asistenciales, cooperación entre los sectores sanitario y social, participación y movilización social. Conclusión: el estado del arte reveló que la atención prenatal, sobre todo construida por relaciones que poco avanzan hacia el logro de la integralidad, termina dificultando la construcción de este principio en las prácticas cotidianas.
\end{abstract}

Palabras clave: Atención primaria de salud; Atención prenatal; Integralidad en salud; Revisión.

\title{
1. Introdução
}

O cuidado pré-natal volta-se à promoção e proteção da saúde de mulheres e crianças, alcance dependente do estabelecimento de relacionamento colaborativo entre mulheres, famílias e profissionais (Who, 2016). No Brasil, o acesso à atenção pré-natal está crescente, porém sua qualidade permanece lacunar (Cecílio \& Reis, 2018; Marsciani, 2014; Santos \& Souza, 2017). Nesta perspectiva, observa-se práticas protocolares e procedimentos preconizados que desconsideram a valorização, tanto da subjetividade quanto da intersubjetividade (Cecílio \& Reis, 2018; Marsciani, 2014; Santos \& Souza, 2017). Neste contexto descreve-se escuta desinteressada (Domingues et al., 2015; Tomasi et al., 2017), fazer burocrático, restrito ao preenchimento de fichas, solicitação de exames e medidas de altura uterina, com insuficiências relacionais, inclusive vivenciadas enquanto violência e repressão (Santos \& Souza, 2017; Goudard et al., 2016).

Em contraposição às incipiências apontadas acima, as indicativas são de adoção da filosofia de cuidado centrado na mulher gestante, reconhecimento dos direitos e rede de apoio social e, promoção do empoderamento a partir de espaços colaborativos e dialógicos (Barger et al., 2015; Mattos, 2005; Butler et al., 2015; McNeill \& Reiger, 2015). Nesta direção, a integralidade, princípio tomado governamentalmente para orientar a atenção à saúde no Brasil, supõe um cuidado à saúde e uma gestão setorial que reconheça a autonomia e a diversidade cultural e social das pessoas e das populações (Mattos, 2005).

Ao considerar os demais princípios assumidos na atenção à saúde, a universalidade impele construir o acesso para todos, o da equidade reivindica pactuar com todos o que cada um necessita e, o da integralidade impulsiona o saber e fazer, o "quê" e "como" para responder universalmente às necessidades de cada um (Ayres, 2004; Ayres, 2009; Ayres et al., 2012). Enfatiza que o encontro no cuidado em saúde precisa ser valorizado e requer olhar individualizado frente ao sujeito, diálogo genuíno, trocas intersubjetivas, no intuito de resgatar o sentido do cuidado em si (McNeill \& Reiger, 2015; Ayres, 2004; Ayres, 2009). Portanto, implica acolher a intersubjetividade em cada encontro, com esforços para apreender e atender a singularidade ali manifesta (Ayres, 2001).

Ao considerar as inadequações do pré-natal brasileiro, e em especial, ao se tomar a integralidade do cuidado (Ayres, 2004; Ayres, 2009; Ayres et al., 2012) e as experiências do cenário internacional como fomentadores dessa discussão, é que se propôs esta revisão cujo objetivo é mapear e refletir o estado da arte da integralidade no cuidado pré-natal na atenção primária.

Este estudo pode contribuir para identificar os fatores que se articulam com a integralidade do cuidado no pré-natal e; discutir os desafios e alcances para sua aplicabilidade na prática da assistência pré-natal desenvolvida na atenção primária à saúde.

\section{Metodologia}

Trata-se de uma revisão de escopo, que visa explorar de forma sistematizada a produção de conhecimento acerca de determinada temática (Peters et al., 2015). O delineamento seguiu o guideline do Instituto Joanna Briggs (JBI) (Peters et al., 
2015). A estrutura do método contém a identificação do título de pesquisa, objetivo da pesquisa e a pergunta da pesquisa, baseada na estrutura mnemônica População, Conceito e Contexto (PCC), estratégia de busca e identificação de estudos relevantes, frente aos critérios de inclusão e exclusão, caracterizando a forma de extração e mapeamento dos resultados, segundo o Preferred Reporting Items for Systematic Reviews and Meta-Analyses (PRISMA).

Nesta revisão, conforme estratégia PCC, para População, gestantes e profissionais de saúde envolvidos com o prénatal; para Conceito, integralidade; e para Contexto, cuidado pré-natal na atenção primária. Desse modo, a questão de revisão estabelecida foi: "Qual o estado da arte sobre a integralidade no cuidado pré-natal na atenção primária?".

Os critérios de elegibilidade dos estudos encontrados foram: estar nos idiomas inglês, espanhol ou português; ser dos tipos primário ou secundário; ter abordagem quantitativa, qualitativa ou ainda quanti qualitativa e/ou mista; conter aspectos da integralidade no pré-natal; e, ter sido publicado no período de 1980, devido marco inicial o ano do Programa de Atenção Integral à Saúde da Mulher (PAISM) (Ministério da Saúde, 1984) a junho de 2019, quando a dissertação de mestrado foi realizada (Barbieri, 2020). Excluíram-se aqueles no formato de carta ao editor, resumos e anais de eventos; sites ou mídias sociais.

Seguindo os critérios do JBI (Peters et al., 2015), identificou-se descritores e palavras chaves para composição das estratégias de busca, as quais foram desenvolvidas, de forma independente, no período de março a julho de 2019, por duas autoras deste estudo. As buscas pelos documentos e publicações na temática envolveram: bases de dados e biblioteca digital: Cumulative Index to Nursing and Allied Health Literature (CINAHL), COCHRANE, Centro Latino-Americano e do Caribe de Informação em Ciências da Saúde (BIREME), PUBMED, Scientific Eletronic Library Online (SCIELO); as plataformas: SCOPUS e Web of Science e o Banco digital de Teses e Dissertações da Capes.

A partir da seleção dos descritores, seus sinônimos e os booleanos, criou-se expressões de busca, de acordo com cada base ou plataforma, mas de modo geral, seguiu-se a estratégia: ("Pregnant" OR "Pregnant Women") AND ("Nurses" OR "medical care" OR "Patient care team" OR "Nursing team") AND ("Prenatal care" AND "Primary health Care") AND ("Quality of health care" AND "Primary health Care") AND ("Perinatal care" OR "Nursing Care") AND ("Quality of health care" AND "comprehensive health care").

$\mathrm{Na}$ etapa de identificação dos estudos, dois revisores independentes realizaram leitura criteriosa do título e resumo, aplicaram os critérios de elegibilidade, para posterior leitura integral, quando as demais autoras foram envolvidas. Diante de impasses ou divergência quanto à manutenção ou exclusão da publicação, um terceiro revisor foi acionado. Ainda, revisões das referências bibliográficas foram desenvolvidas com vistas a localização de materiais (Peters et al., 2015).

Foram recuperados 1.848 estudos, dos quais 114 estudos estavam duplicados. A exploração dos títulos e resumos repercutiu em 269 estudos potencialmente elegíveis, dos quais 142 foram integralmente lidos. Após esta leitura, excluiu-se 116 publicações, permanecendo 24. As referências deles foram apreciadas, 31 aparentavam atender a esta revisão, contudo aplicado o processo de elegibilidade, excluiu-se 29. No total, 26 publicações integraram a revisão. A Figura 1 pormenoriza o processo de seleção dos estudos tomando o PRISMA adaptado para Scoping reviews (Peters et al., 2015). 
Figura 1 - Esquema do processo de extração dos dados. São Carlos, São Paulo, Brasil 2021.

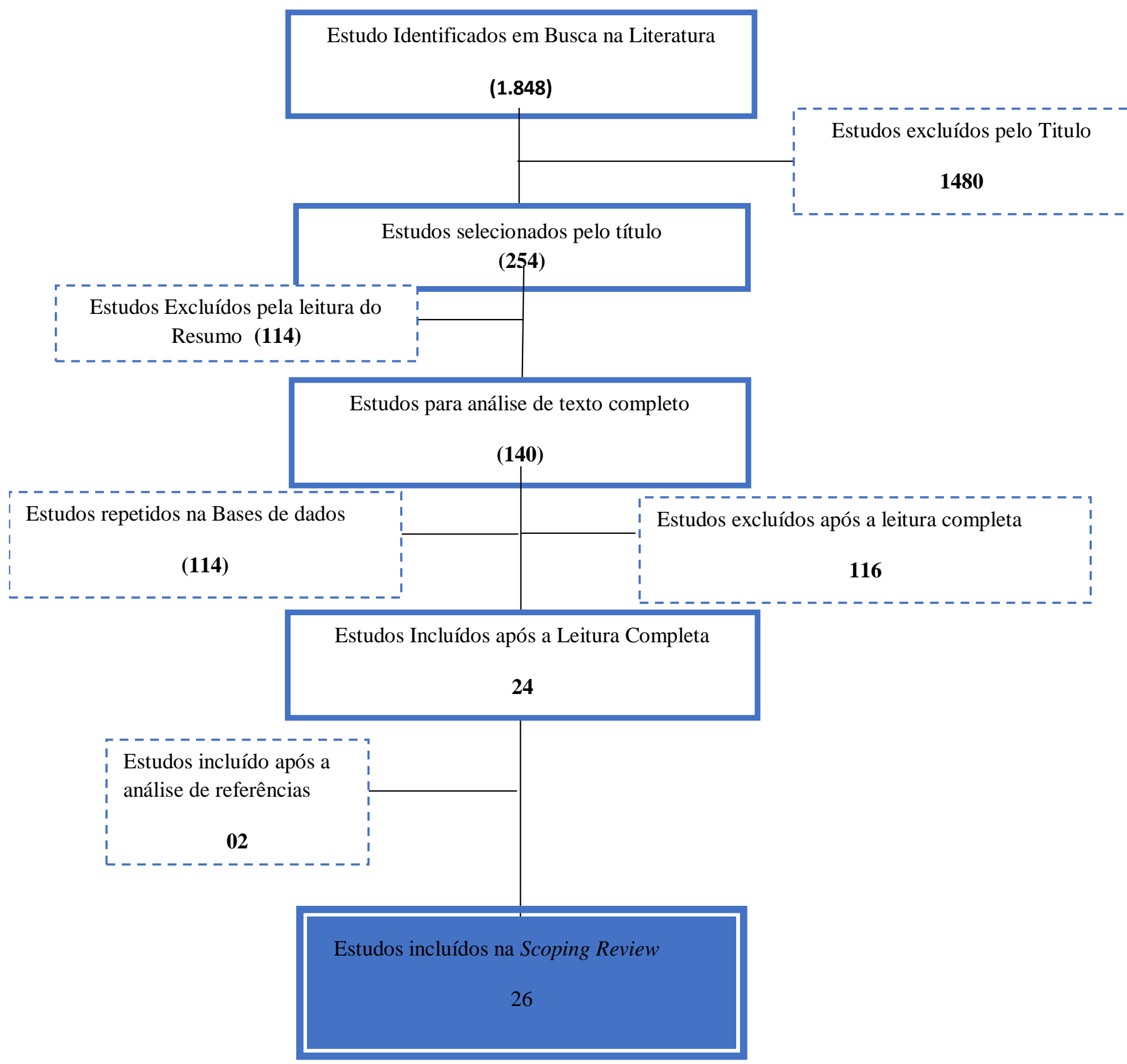

Fonte: Prisma (ScR) — Fluxograma para scoping review (Peters et al., 2015).

Para extração dos dados, utilizou-se um formulário estruturado, conforme as orientações do JBI (Peters et al., 2015), e extraiu-se os dados relativos ao: título do estudo, local do estudo, ano de publicação, título do periódico, tipo de pesquisa, objetivo da pesquisa, abordagem teórico-metodológica, população investigada e principais resultados e elementos correlacionados ao conceito da integralidade do cuidado. Dados esses apresentados de maneira descritiva e em quadros.

Ainda, reitera-se que, os principais resultados e os dados relacionados ao conceito da integralidade do cuidado foram discutidos a partir de categorias conceituais estabelecidas e intituladas em consonância com os quatro eixos da Integralidade na propositura de Ayres, ou seja, das necessidades, finalidades, articulações e interações (Ayres, 2009), especificamente Eixo 1 - 
Necessidades de saúde das gestantes; Eixo 2 - Finalidades do trabalho em saúde com gestantes, Eixo 3 - Articulação entre saberes e ações no cuidado de gestantes e Eixo 4 - Interação entre sujeitos no cotidiano do trabalho com gestantes.

\section{Resultados e Discussão}

Dos 26 estudos selecionados, 20 são datados da segunda década dos anos 2000, sendo quatro de 2015, quatro de 2017, três referentes à 2018 e três de 2019.

Em relação ao local e país dos estudos, 17 são publicações nacionais e nove internacionais, distribuídas respectivamente pelos países: Estados Unidos, Argentina, Cuba, Arábia Saudita, Tailândia, África do Sul, Irlanda e Austrália. Quanto à abordagem/método, 14 estudos são de enfoque qualitativo, sete quantitativos descritivos, três de revisão e dois quantiqualitativos. Em relação a amostra ou a população investigada, identificaram-se dez estudos com mulheres, cinco envolveram mulheres e equipe de saúde (médico, enfermeiro, parteiras) e quatro apenas profissionais de saúde (enfermeiro, médico, agente comunitário de saúde, dentista).

O Quadro 1 traz dados dos estudos selecionados, informações como autoria, ano, país de estudo, título e os elementos de integralidade em saúde foram extraídos dos estudos e identificados no quadro

Quadro 1 - Caracterização dos estudos quanto a Autoria, Ano, País, Títulos e Elementos de Integralidade. São Carlos, São Paulo, Brasil 2021.

\begin{tabular}{|c|c|c|c|}
\hline Autoria/Ano & $\begin{array}{l}\text { País do } \\
\text { estudo }\end{array}$ & Título & Elementos de integralidade \\
\hline $\begin{array}{l}\text { Lazarus } \quad \& \\
\text { Philipson, } 1990\end{array}$ & $\begin{array}{l}\text { Estados } \\
\text { Unidos }\end{array}$ & $\begin{array}{l}\text { A longitudinal Study Comparing the } \\
\text { Prenatal Care of Puerto Rican and } \\
\text { White Woman }\end{array}$ & $\begin{array}{l}\text { Continuidade do cuidado: organização dos serviços, segundo as } \\
\text { necessidades das gestantes }\end{array}$ \\
\hline $\begin{array}{l}\text { Langer et al., } \\
2002\end{array}$ & $\begin{array}{l}\text { Argentina, } \\
\text { Cuba, } \\
\text { Arábia } \\
\text { Saudita e } \\
\text { Tailândia }\end{array}$ & $\begin{array}{l}\text { Are women and providers satisfied } \\
\text { with antenatal care? Views on a } \\
\text { standard and a simplified, evidence- } \\
\text { based model of care in four developing } \\
\text { countries }\end{array}$ & $\begin{array}{l}\text { Cuidado construído pela maior interação e reconhecimento das } \\
\text { opiniões das gestantes }\end{array}$ \\
\hline $\begin{array}{l}\text { Delfino et al., } \\
2004\end{array}$ & Brasil & $\begin{array}{l}\text { O processo de cuidar participante com } \\
\text { um grupo de gestantes: repercussões na } \\
\text { saúde integral individual-coletiva }\end{array}$ & $\begin{array}{l}\text { Cuidado pautado em uma abordagem dialógica, integral em } \\
\text { momentos individuais e coletivos }\end{array}$ \\
\hline $\begin{array}{l}\text { Rios \& Vieira, } \\
2007\end{array}$ & Brasil & $\begin{array}{l}\text { Ações educativas no pré-natal: reflexão } \\
\text { sobre a consulta de enfermagem como } \\
\text { um espaço para educação em saúde }\end{array}$ & $\begin{array}{l}\text { Cuidado que valorize a individualidade dos sentimentos, } \\
\text { emoções, mudanças físicas e necessidades de aprendizados das } \\
\text { gestantes }\end{array}$ \\
\hline $\begin{array}{l}\text { Parada } \& \\
\text { Tonete, } 2002\end{array}$ & Brasil & $\begin{array}{l}\text { O cuidado em saúde no ciclo gravídico } \\
\text { puerperal sob a perspectiva de usuárias } \\
\text { de serviços públicos }\end{array}$ & $\begin{array}{l}\text { Prevenção de agravos, compromisso com a qualidade de vida, } \\
\text { autonomia e humanização da gestação, e valorização da } \\
\text { participação comunitária }\end{array}$ \\
\hline $\begin{array}{l}\text { Mathibe-Neke, } \\
2008\end{array}$ & $\begin{array}{l}\text { África do } \\
\text { Sul }\end{array}$ & $\begin{array}{l}\text { The expectations of pregnant women } \\
\text { regarding antenatal care }\end{array}$ & $\begin{array}{l}\text { Cuidados de saúde considerados direito humano básico e } \\
\text { empoderamento das mulheres }\end{array}$ \\
\hline Santos, 2009 & Brasil & $\begin{array}{l}\text { Integralidade do cuidado à gestante, } \\
\text { puérpera e recém-nascido: o olhar de } \\
\text { usuárias }\end{array}$ & $\begin{array}{l}\text { Conjunto articulado e contínuo de ações e serviços preventivos e } \\
\text { curativos, individuais e coletivos; dispositivo legal-institucional, } \\
\text { portador de valores ético-políticos, que considera o cuidado } \\
\text { como atividade humana }\end{array}$ \\
\hline Novick, 2009 & $\begin{array}{l}\text { Estados } \\
\text { Unidos }\end{array}$ & $\begin{array}{l}\text { Women's experience of prenatal care: } \\
\text { an integrative review }\end{array}$ & $\begin{array}{l}\text { Cuidado centrado no Pré-natal, que busca a participação ativa do } \\
\text { profissional e dos companheiros nas formas de cuidado para } \\
\text { atingir as necessidades das gestantes }\end{array}$ \\
\hline
\end{tabular}




\begin{tabular}{|c|c|c|c|}
\hline $\begin{array}{l}\text { Zampieri } \quad \& \\
\text { Erdmann, } 2010\end{array}$ & Brasil & $\begin{array}{l}\text { Cuidado humanizado no pré-natal: um } \\
\text { olhar para além das divergências e } \\
\text { convergências }\end{array}$ & $\begin{array}{l}\text { Fruto do esforço e da confluência dos vários saberes de uma } \\
\text { equipe multiprofissional para atender as necessidades de saúde, } \\
\text { sendo plena quando há articulação entre os serviços de saúde e } \\
\text { com outras instituições sociais }\end{array}$ \\
\hline $\begin{array}{l}\text { Hanson et al., } \\
2009\end{array}$ & $\begin{array}{l}\text { Estados } \\
\text { Unidos }\end{array}$ & $\begin{array}{l}\text { A Critical Appraisal of Guidelines for } \\
\text { Antenatal Care: Components of Care } \\
\text { and Priorities in Prenatal Education }\end{array}$ & $\begin{array}{l}\text { Cuidado centrado na mulher, evidenciando necessidades de } \\
\text { conforto, bem-estar e impactos do cuidado para a família }\end{array}$ \\
\hline Duarte, 2012 & Brasil & $\begin{array}{l}\text { Motivos que levam as gestantes a } \\
\text { fazerem o pré-natal: um estudo das } \\
\text { representações sociais }\end{array}$ & $\begin{array}{l}\text { Comunicação como relação terapêutica, acolhimento e vínculo } \\
\text { entre gestantes/profissionais }\end{array}$ \\
\hline $\begin{array}{l}\text { Philippi et al., } \\
2014\end{array}$ & $\begin{array}{l}\text { Estados } \\
\text { Unidos }\end{array}$ & $\begin{array}{l}\text { Facilitators of prenatal care access in } \\
\text { rural Appalachia }\end{array}$ & $\begin{array}{l}\text { Características dos profissionais, a atenção com os usuários no } \\
\text { cuidado individualizado, consultas dialogadas, troca de } \\
\text { conhecimentos e melhoria do vínculo com o profissional }\end{array}$ \\
\hline Sodré, 2015 & Brasil & $\begin{array}{l}\text { Atenção básica ao pré-natal e puerpério } \\
\text { no estado de Goiás }\end{array}$ & $\begin{array}{l}\text { Acessibilidade e acolhimento dos usuários em uma lógica } \\
\text { organizacional. Boa estrutura dos serviços de saúde, adequadas } \\
\text { inter-relações pessoais entre profissionais e gestores do sistema, } \\
\text { disponibilização de serviços de atenção à mulher. Correção de } \\
\text { dificuldades no acesso ao atendimento e agendamento correto } \\
\text { das consultas }\end{array}$ \\
\hline Silva, 2015 & Brasil & $\begin{array}{l}\text { Assistência pré-natal na rede municipal } \\
\text { de Niterói: a ótica valorativa dos } \\
\text { profissionais de saúde }\end{array}$ & $\begin{array}{l}\text { Preocupação com o cuidado integral das gestantes, vínculo, } \\
\text { acolhimento, simpatia dos profissionais e inclusão da família no } \\
\text { cuidado. }\end{array}$ \\
\hline $\begin{array}{l}\text { Baxley \& } \\
\text { Ibitayo, } 2015\end{array}$ & $\begin{array}{l}\text { Estados } \\
\text { Unidos }\end{array}$ & $\begin{array}{l}\text { Expectations of Pregnant Women of } \\
\text { Mexican Origin Regarding Their } \\
\text { Health Care Providers }\end{array}$ & $\begin{array}{l}\text { Cuidado pautado na confiança e comunicação visando atingir o } \\
\text { cuidado centrado na pessoa }\end{array}$ \\
\hline $\begin{array}{l}\text { Butler et al., } \\
2015\end{array}$ & Irlanda & $\begin{array}{l}\text { Evaluating midwife-led antenatal care: } \\
\text { Choice, experience, effectiveness, and } \\
\text { preparation for pregnancy }\end{array}$ & $\begin{array}{l}\text { Elementos que demonstram uma melhor atenção pré-natal guiada } \\
\text { por parteiras do que em outros modelos, com ênfase na } \\
\text { continuidade do cuidado e experiência vivenciada pelas mulheres }\end{array}$ \\
\hline $\begin{array}{l}\text { Nogueira et al., } \\
2017\end{array}$ & Brasil & $\begin{array}{l}\text { Prenatal care and practices developed } \\
\text { by the health team: integrative review }\end{array}$ & $\begin{array}{l}\text { Soma de saberes na atuação em equipe para a construção de } \\
\text { respostas efetivas e eficazes aos problemas de saúde e na } \\
\text { produção de um cuidado integral, visando melhorias na } \\
\text { qualidade de vida das usuárias }\end{array}$ \\
\hline $\begin{array}{l}\text { Valdes et al., } \\
2017\end{array}$ & Brasil & $\begin{array}{l}\text { Programa mais médicos: qualificação } \\
\text { da atenção ao pré-natal e puerpério no } \\
\text { âmbito da estratégia de saúde da } \\
\text { família }\end{array}$ & $\begin{array}{l}\text { Melhorias na organização do trabalho em saúde, início precoce e } \\
\text { ampla cobertura do pré-natal, ações de promoção com vistas a } \\
\text { uma assistência integral }\end{array}$ \\
\hline Costa, 2017 & Brasil & $\begin{array}{l}\text { Avaliação da qualidade da atenção } \\
\text { primária à saúde para a Rede Cegonha }\end{array}$ & $\begin{array}{l}\text { Necessidade de serviços preventivos e curativos, para além dos } \\
\text { aspectos biológicos }\end{array}$ \\
\hline $\begin{array}{l}\text { Prudêncio, } \\
2017\end{array}$ & Brasil & $\begin{array}{l}\text { Avaliação da expectativa e satisfação } \\
\text { da gestante com o cuidado pré-natal na } \\
\text { Atenção Primária à Saúde }\end{array}$ & $\begin{array}{l}\text { Preparo clínico e científico na busca da resolutividade ampliada } \\
\text { na assistência à gestante, família e comunidade, compreendendo } \\
\text { a situação socioeconômica e cultural. Promoção da autonomia da } \\
\text { mulher, a qualidade da atenção embasada na escuta ativa e no } \\
\text { desempenho satisfatório do profissional. }\end{array}$ \\
\hline $\begin{array}{l}\text { Warmling et } \\
\text { al., } 2018\end{array}$ & Brasil & $\begin{array}{l}\text { Práticas sociais de medicalização \& } \\
\text { humanização no cuidado de mulheres } \\
\text { na gestação }\end{array}$ & $\begin{array}{l}\text { O processo de medicalização não está restrito apenas ao tipo de } \\
\text { parto, mas também aos programas de pré-natal, que contabilizam } \\
\text { mais exames do que interação entre médico-cliente. A atenção } \\
\text { humanizada à mulher na gestação e no parto deve incorporar o } \\
\text { caráter biopsicossocial do cuidado }\end{array}$ \\
\hline $\begin{array}{l}\text { Guimarães et } \\
\text { al., } 2010\end{array}$ & Brasil & $\begin{array}{l}\text { Acesso e qualidade da atenção pré- } \\
\text { natal na Estratégia Saúde da Família: } \\
\text { infraestrutura, cuidado e gestão }\end{array}$ & $\begin{array}{l}\text { Avaliação organizacional dos serviços do pré-natal, nas } \\
\text { subdimensões: gerenciais, acesso e qualidade do pré-natal }\end{array}$ \\
\hline Wright et al., & Austrália & Exploring routine hospital antenatal & Cuidado centrado na mulher, valorizando a interação entre \\
\hline
\end{tabular}




\begin{tabular}{|l|l|l|l|}
\hline 2018 & & $\begin{array}{l}\text { care consultations-Anethnographic } \\
\text { study }\end{array}$ & $\begin{array}{l}\text { mulher e parteira, para além dos protocolos e documentos } \\
\text { físicos. Mulher como protagonista do processo, atendendo suas } \\
\text { necessidades, explicando a elas os conteúdos essenciais. }\end{array}$ \\
\hline $\begin{array}{l}\text { et Livramento } \\
\text { eardoso, 2018 }\end{array}$ & Brasil & $\begin{array}{l}\text { Perceptions of pregnant women about } \\
\text { prenatal care in primary health care }\end{array}$ & $\begin{array}{l}\text { Acolhimento e reconhecimento das necessidades das gestantes, } \\
\text { visando o estabelecimento de vínculos }\end{array}$ \\
\hline $\begin{array}{l}\text { Cunha et al., } \\
\text { Serviços de Atenção Básica à Saúde do } \\
\text { Piaú no contexto do Programa } \\
\text { Nacional de Melhoria do Acesso e da } \\
\text { Qualidade da Atenção Básica }\end{array}$ & $\begin{array}{l}\text { Conjunto de recursos disponíveis aos profissionais para } \\
\text { possibilitar atender ao máximo as necessidades dos usuários, } \\
\text { ofertar e reconhecer "adequadamente problemas funcionais, } \\
\text { orgânicos ou sociais" }\end{array}$ \\
\hline $\begin{array}{l}\text { Evaluation of prenatal care in Primary } \\
\text { Health Care in Brazil }\end{array}$ & $\begin{array}{l}\text { Aspectos estruturais e operacionais devem ser garantidos para } \\
\text { monitoramento contínuo e de qualidade do atendimento } \\
\text { humanizado. }\end{array}$ \\
\hline
\end{tabular}

Fonte: Dados de pesquisa (2021).

A seguir, apresenta-se o Quadro 2 com análise dos eixos propostos por Ayres (2009), de acordo com cada estudo selecionado nesta revisão, mostrando os alcances e desafios frente a integralidade do cuidado para cada eixo: eixo das necessidades, eixo das finalidades, eixo da articulação e eixo das interações.

Quadro 2 - Apresentação das categorias conceituais, número de estudos selecionados e síntese dos resultados com base nos alcances e desafios, segundo a integralidade de Ayres. São Carlos, São Paulo, Brasil 2021.

\begin{tabular}{|c|c|c|}
\hline $\begin{array}{l}\text { Categorias } \\
\text { Conceituais }\end{array}$ & Estudos relacionados & Síntese dos resultados segundo a Integralidade de Ayres \\
\hline $\begin{array}{l}\text { Eixo } 1 \text { - Necessidades } \\
\text { de saúde das gestantes }\end{array}$ & $\begin{array}{l}\text { N=16 } \\
\text { Lazarus \& Philipson, 1990; Langer et al., } \\
\text { 2002; Delfino et al., 2004; Rios \& Vieira, } \\
\text { 2007; Parada \& Tonete, 2002; Santos, 2009; } \\
\text { Novick, 2009; Zampieri \& Erdmann, 2010; } \\
\text { Duarte, 2012; Philippi et al., 2014; Sodré, } \\
\text { 2015; Silva, 2015; Valdes et al., 2017; } \\
\text { Wright et al., 2018; Livramento et al., 2019. }\end{array}$ & $\begin{array}{l}\text { O apoio informacional e educativo obtido junto aos } \\
\text { profissionais de saúde esteve destacado pelas gestantes, assim } \\
\text { como o atitudinal do profissional, com ênfase à simpatia e } \\
\text { educação. } \\
\text { Ainda, a abordagem dialógica e sua relação com o atendimento } \\
\text { das necessidades das gestantes esteve pautado, assinalando } \\
\text { rodas de conversa, ações dinâmicas e em grupos como de } \\
\text { contribuição. }\end{array}$ \\
\hline $\begin{array}{l}\text { Eixo } 2 \text { - Finalidades do } \\
\text { trabalho em saúde com } \\
\text { gestantes }\end{array}$ & $\begin{array}{l}\mathrm{N}=8 \\
\text { Langer et al., 2002; Delfino et al., 2004; Rios } \\
\& \text { Vieira, 2007; Mathibe-Neke, 2008; Santos, } \\
\text { 2009; Novick, 2009; Zampieri \& Erdmann, } \\
\text { 2010; Warmling et al., 2018. }\end{array}$ & $\begin{array}{l}\text { A relação interpessoal e sua relação com escuta qualificada e } \\
\text { acolhimento foi assinalada, assim como a presença de } \\
\text { desigualdade em saúde e discriminação. Ainda, destacaram-se } \\
\text { incipiências para integrar a família ao Pré-natal (PN). }\end{array}$ \\
\hline $\begin{array}{l}\text { Eixo } 3-\text { Articulação } \\
\text { entre saberes e aços no } \\
\text { cuidado de gestantes }\end{array}$ & $\begin{array}{l}\text { N=16 } \\
\text { Lazarus \& Philipson, 1990; Langer et al., } \\
\text { 2002; Delfino et al., 2004; Rios \& Vieira, } \\
\text { 2007; Mathibe-Neke, 2008; Santos, 2009; } \\
\text { Zampieri \& Erdmann, 2010; Sodré, 2015; } \\
\text { Silva, 2015; Butler et al., 2015; Nogueira et } \\
\text { al., 2017; Valdes et al., 2017; Costa, 2017; } \\
\text { Warmling et al., 2018; Guimarães et al., } \\
\text { 2010; Cardoso, 2018. }\end{array}$ & $\begin{array}{l}\text { Vivências empíricas e científicas no processo ensino- } \\
\text { aprendizagem entre as equipes responde às necessidades da } \\
\text { comunidade. O cenário internacional recomenda treinamento de } \\
\text { médicos com parteiras visando um melhor entendimento das } \\
\text { necessidades das gestantes. A demora para as gestantes serem } \\
\text { atendidas e a falta de articulação pela deficiência de recursos } \\
\text { materiais e humanos são problemáticas nacional e } \\
\text { internacional. }\end{array}$ \\
\hline $\begin{array}{l}\text { Eixo } 4 \text { - Interação entre } \\
\text { sujeitos no cotidiano do } \\
\text { trabalho com gestantes }\end{array}$ & $\begin{array}{l}\text { N =5 } \\
\text { Delfino et al., 2004; Rios \& Vieira, 2007; } \\
\text { Santos, 2009; Zampieri \& Erdmann, 2010; } \\
\text { Valdes et al., 2017. }\end{array}$ & $\begin{array}{l}\text { Necessidade de apostas no planejamento do PN, reorganização } \\
\text { de protocolos e documentações, com valorização das trocas de } \\
\text { saberes, acolhimento, vínculo e a centralidade das necessidades } \\
\text { da gestante no desenvolvimento do cuidado. }\end{array}$ \\
\hline
\end{tabular}


A revisão efetuada permitiu encontrar que a maioria dos estudos compreende a integralidade como ações voltadas para a interação entre profissionais, usuários e serviços de saúde. Evidencia-se o reconhecimento da perspectiva dialógica, da valorização da singularidade, autonomia, empatia, acessibilidade, acolhimento, prevenção, tratamento, cuidado contínuo e centrado na mulher gestante (Lazarus \& Philipson, 1990; Langer et al., 2002; Delfino et al., 2004; Rios \& Vieira, 2007; Parada \& Tonete, 2002; Santos, 2009; Novick, 2009; Zampieri \& Erdmann, 2010; Hanson et al., 2009; Duarte, 2012; Philippi et al., 2014; Sodré, 2015; Silva, 2015; Baxley \& Ibitayo, 2015; Nogueira et al., 2017; Costa, 2017; Prudêncio, 2017; Warmling et al., 2018; Guimarães et al., 2010; Wright et al., 2018; Livramento et al., 2019; Cardoso, 2018; Cunha et al., 2019) enquanto partes que integram o cuidado integral.

Salienta-se que apenas três estudos (Mathibe-Neke, 2008; Butler et al., 2015; Valdes et al., 2017) não trouxeram explicitamente o termo integralidade, no entanto, ressaltaram elementos que podem potencializar o alcance para tal, a saber: cuidado enquanto direito humano básico, de empoderamento feminino e de melhorias na organização, cobertura e assistência pré-natal. Ademais, estudos internacionais (Lazarus \& Philipson, 1990; Langer et al., 2002, Novick, 2009; Hanson et al., 2009; Wright et al., 2018) não utilizaram o termo integralidade, mas trouxeram elementos consonantes ao conceito, como reconhecimento da mulher e sua experiência singular, a exemplo do Pregnancy Century (Cuidado Centrado na gestante) e Collective Prenatal (Pré-natal coletivo).

$\mathrm{Na}$ avaliação do eixo das necessidades, o qual se refere à qualidade da escuta, acolhimento e resposta às demandas de atenção à saúde, para além das necessidades biológicas e dos distúrbios morfológicos ou funcionais (Ayres, 2009), mostrou que a avaliação positiva da atenção ao pré-natal por parte das gestantes se dá quando são atendidas por profissionais que denotam interesse, se mostram disponíveis e abertos, e possibilitam uma avaliação física com concomitaante compartilhamento das ações cuidativas às mesmas (Lazarus \& Philipson, 1990; Langer et al., 2002; Santos, 2009; Duarte, 2012; Wright et al., 2018).

Estudo irlandês (Butler et al., 2015) sobre o cuidado prestado por parteiras assinalou a importância do tempo das consultas e sua relação com o acolhimento de necessidades das gestantes. No estudo australiano (Wright et al., 2018) mostrouse que ser chamada pelo nome, ter um atendimento disponível e aberto às perguntas sob suporte de material educativo, teve alcances positivos. Estes resultados reforçam a importância e a necessidade da escuta qualificada, diálogo e acolhimento (Delfino et al., 2004; Santos, 2009; Warmling et al., 2018).

Ainda que a presente revisão evidencie alcances no eixo das necessidades, os desafios seguem existindo. O modelo de atenção pré-natal em vigência, a nível global, persiste seguindo o paradigma biomédico (Lazarus \& Philipson, 1990; Langer et al., 2002; Parada \& Tonete, 2002; Mathibe-Neke, 2008; Zampieri \& Erdmann, 2010; Sodré, 2015; Silva, 2015; Nogueira et al., 2017; Cunha et al., 2019), que vai na contramão da subjetividade e da valoração da mulher enquanto protagonista do cuidado, influenciando diretamente a integralidade.

A superação do domínio e superioridade técnica do profissional precisa ser transposta, com apostas no diálogo, na corresponsabilidade, na construção intersubjetiva que perpassa a valorização de subjetividades. Incorporar tais premissas desfavorecem ocorrências como falta de atenção e paciência na escuta, falta de interação e comunicação, falas ofensivas ou discriminatórias (Lazarus \& Philipson, 1990; Langer et al., 2002; Parada \& Tonete, 2002; Mathibe-Neke, 2008), e podem caminhar para o alcance de práticas mais integrais no cuidado pré-natal.

Seguindo na mesma premissa, o eixo das finalidades que, remete aos modos de integração das ações de promoção e prevenção de saúde e agravos, tratamento e recuperação da saúde/reinserção social, criação de estratégias de articulação no desenvolvimento das ações (Sodré, 2015), revelou grandes desafios (Langer et al., 2002; Rios \& Vieira, 2007; Mathibe-Neke, 2008; Novick, 2009; Warmling et al., 2018).

O processo de trabalho efetivou iatrogenias e danos, como demora dos resultados de exames, falta de local que 
ofereça serviços laboratoriais, escassez estrutural para a execução dos trabalhos, déficit nos prontuários eletrônicos e até mesmo conflitos com os prontuários em papel, falta de recursos materiais e humanos, falhas na integração e comunicação entre a coordenação e gestores de serviços de saúde, que atingem a intersetorialidade entre os serviços de referência e contra referência (Lazarus \& Philipson, 1990; Langer et al., 2002; Rios \& Vieira, 2007; Parada \& Tonete, 2002; Mathibe-Neke, 2008; Santos, 2009; Zampieri \& Erdmann, 2010; Sodré, 2015; Silva, 2015; Butler et al., 2015; Nogueira et al., 2017; Valdes et al., 2017; Costa, 2017; Warmling et al., 2018; Guimarães et al., 2010; Cardoso, 2018).

O cuidado centrado na gestante e na família, atento às articulações entre ações de promoção da saúde, prevenção de agravos, tratamento de doenças, sensível aos sofrimentos e recuperação da saúde/reinserção social, implicam no envolvimento dos indivíduos, famílias e comunidades nas ações, ou sua exclusão ou segmentação (Philippi et al., 2014; Sodré, 2015). O cuidado em saúde ainda se mostra pautado em desigualdades que estigmatizam, hierarquizam e tornam as ações de cuidado pouco efetivas.

Ações que apostam em atividades educativas com linguajar acessível e partilhado, bem como grupos e vivências que promovam empoderamento da mulher e da família e outras estratégias de cunho coletivo são exemplos de alcances (Langer et al., 2002; Delfino et al., 2004; Rios \& Vieira, 2007; Mathibe-Neke, 2008; Santos, 2009; Zampieri \& Erdmann, 2010; Warmling et al., 2018).

Neste olhar, destaca-se uma publicação brasileira que indica a necessidade de treinamentos e capacitações que promovam o trabalho em equipe e qualifiquem o pré-natal (Valdes et al., 2017). Embora políticas públicas nacionais como a Estratégia da Saúde da Família (Barbiani et al., 2016) venham apresentando bons resultados quanto ações multiprofissionais, faz-se fundamental empregar na atenção básica os fundamentos das políticas nacionais direcionadoras do pré-natal, para tentar alcançar qualidade no cuidado, tornando-o menos fragmentado, com gestão que propiciem um trabalho sem excesso de funções e rotatividade de setores, diminuição de falhas na comunicação interna e entre os serviços da rede e equipes, uma vez que essas ações são contrárias a integralidade e atrapalham o alcance dos eixos da finalidade (Silva et al., 2014; Dias et al., 2014; Protassio et al., 2014; Martinelli et al., 2014).

Ao dar sequência para o eixo das articulações, o qual abarca a composição de saberes interdisciplinares, equipes multiprofissionais e ações intersetoriais no desenvolvimento das ações e estratégias de atenção à saúde (Ayres, 2009) observou-se nesta revisão a relevância das trocas de experiências em grupo para aprendizagens (Delfino et al., 2004, Valdes et al., 2017; Costa, 2017).

Individualizar o cuidado diante dos sentimentos e vivências das mulheres e o trabalho em equipe, na qual o enfermeiro estava no comando, contribuíram para o cuidado integral e longitudinal (Santos, 2009). De modo geral, a maior parte das publicações que trouxeram resultados frente a este eixo buscaram demonstrar o valor do cuidado colaborativo, dos saberes e práticas multi, Inter ou transdisciplinares como enfrentamento para o cuidado fragmentado observado em vários serviços de saúde, e também na atenção ao pré-natal, em especial, no Brasil (Langer et al., 2002; Delfino et al., 2004; Santos, 2009; Zampieri \& Erdmann, 2010; Sodré, 2015; Silva, 2015; Butler et al., 2015; Nogueira et al., 2017; Valdes et al., 2017; Costa, 2017).

Assim sendo, a falta de comunicação interprofissional e interpessoal, em especial, prejudica a articulação dos saberes (Langer et al., 2002; Delfino et al., 2004; Zampieri \& Erdmann, 2010; Warmling et al., 2018; Guimarães et al., 2010), e quase sempre contribui para informações lacunares em prontuário (Lazarus \& Philipson, 1990; Langer et al., 2002; Rios \& Vieira, 2007; Zampieri \& Erdmann, 2010; Hanson et al., 2009; Sodré, 2015; Costa, 2017; Guimarães et al., 2010). Logo, visualiza-se por meio dessa discussão que, se um dos eixos estudados por Ayres (2009) apresenta-se enfraquecido, comumente, observamse reflexos, quase sempre negativos, nos demais eixos.

Neste sentido, Ayres (2009) propõe o eixo das interações, que se refere à qualidade e natureza das interações 
intersubjetivas no cotidiano das práticas de cuidado, construção de relações dialógicas entre os sujeitos dos encontros relacionados à atenção à saúde (Ayres, 2004; Ayres et al., 2012).

Nesta revisão apresentou-se com mais desafios do que alcances atingidos, pois apenas duas publicações (Delfino et al., 2004, Valdes et al., 2017) relavaram ações que envolvem: assistência centrada nas necessidades dos clientes, equipe que acolhe e trabalha demandas individuais e coletivas, além de qualificar as interações intersubjetivas no cotidiano das práticas de cuidado ao criar espaços de discussões e trocas de saberes, o que favorece relações dialógicas entre os sujeitos e melhores resultados quanto à integralidade do cuidado.

Estudo nacional (Valdes et al., 2017) ainda evidencia que o planejamento e a organização dos impressos, protocolos, prontuários tendem a melhorar e facilitar a rotina de trabalho, logo, colabora para agilidade das atividades, contribuindo para maior atenção às demandas das mulheres grávidas e familiares, possibilitando também aos profissionais atualização segundo protocolos institucionais e governamentais, e trabalho em equipes multiprofissionais. Fato este que permite aproximar-se do conceito da integralidade do cuidado proposto por Ayres (Ayres, 2004; Ayres, 2009; Ayres et al., 2012).

No entanto, mesmo com resultados promissores a serem atingidos ao aprofundar-se nos resultados visualizados neste eixo, os desafios (Zampieri \& Erdmann, 2010; Valdes et al., 2017) quanto à reorganização social, ações políticas, mudanças na postura profissional, articulação de redes de atenção à saúde, cooperação intersetorial, além da participação e mobilização social acabam sendo obstáculos reais que precisam ser vencidos para que se estabeleça um cuidado alinhado com a integralidade (Ayres, 2009).

Nesse contexto, a síntese dos resultados apresentados nesta revisão, remete alguns questionamentos: Como reabilitar e promover a saúde materna, através de um modelo de atenção à saúde com uma abordagem interpessoal, indiferente e preconceituosa? Os profissionais estão promovendo, protegendo, recuperando e reabilitando a saúde? As ações estão centradas nas gestantes? As ações estão articuladas? As ações e estratégias de atenção à saúde que considerem a composição de saberes interdisciplinares, equipes multiprofissionais e ações intersetoriais são as que trazem melhores resultados por conseguirem responder, de forma efetiva, as demandas das gestantes e familiares (Silva et al., 2014).

As limitações frente aos desafios implicam em rever os processos de trabalho que burocratizam a assistência, bem como a rotatividade de profissionais, muitas vezes descomprometidos com o cuidado. A falta de recursos materiais e humanos, falhas na comunicação entre a coordenação, gestores e equipes, estrutura inadequada, poucos computadores na atenção primária, déficit nos prontuários eletrônicos, dificuldades na referência e contra referência continuam a ser desafios no eixo das articulações (Dias et al., 2014).

A falta de articulação frente as mudanças de gestão, de setores e excesso de funções são limitações que prejudicam as relações humanas. A nível nacional, houve um aumento da cobertura do acesso ao pré-natal, porém com cuidado inadequado e desigual. Destaca-se a importância de repensar formas de implementar as políticas de saúde pública existentes, visando reduzir as desigualdades. A Rede Cegonha (Ministério da Saúde, 2011) e o cuidado pautado na Política de Humanização do Pré-natal e Nascimento (Ministério da Saúde, 2000) são ações governamentais e nacionais que auxiliam a traçar caminhos para o alcance da integralidade e deve ser incentivada nos cenários de cuidado pré-natal (Protasio et al., 2014).

Por fim, os modelos de atenção pré-natal precisam ser transformados sob horizontes que considerem singularidades, com o da integralidade. É premente repensar questões como processo saúde versus doença, cuidado humanizado versus medicalizado, do contrário é distante ponderar integralidade, objetivar projetos de felicidade e melhorias de desfechos perinatais (Tomasi et al., 2017; Ayres, 2009; Warmling et al., 2018; Leal et al;. 2018). A valorização das interaç̃̃es intersubjetivas no cotidiano das práticas de cuidado perpassa ressignificar, tanto a formação profissional como as práticas em saúde. Um olhar que precisa ser construído a partir dos encontros. 


\section{Considerações Finais}

O estado da arte revelado por esse estudo demonstrou que o cuidado pré-natal ainda está edificado pelas relações que pouco se movem para o alcance da integralidade. Dessa forma, a construção deste princípio apresenta limitações em seu alcance. A categoria que mais contemplou os alcances da integralidade do cuidado foi o eixo das necessidades e, ao mesmo tempo é o que mais aponta desafios. As dificuldades na articulação de saberes e práticas interdisciplinares revelaram necessidades de mudanças na formação dos profissionais de saúde, capacitações e treinamentos no ambiente de trabalho. Ambas as categorias destacam a importância da qualidade das interações intersubjetivas na construção do cuidado e do trabalho em equipe.

As demais categorias estão presentes em menos estudos e evidenciam a limitação dos mesmos para avaliar a intersubjetividade na percepção das usuárias e dos profissionais. Dado que não é uma medida numérica, implica rever práticas de saúde e atitudinais que demonstrem um atendimento de qualidade, desde o acolhimento digno, respeitoso, assegurando o direito à saúde, respeitando a mulheres, independentemente de sua cultura, condições financeiras, psíquicas, valorizando suas necessidades, de forma que a gestante e sua família possam ser atendidas conforme suas prioridades e necessidades e uma relação de cuidado possa ser construída a partir deste encontro.

Muitos estudos nacionais não foram elegíveis, pois, no Brasil, ainda se vincula a qualidade do pré-natal ao número de consultas, início precoce e realização de exames. Por isso, talvez os estudos qualitativos tenham subsidiado os resultados e a discussão em maior escala, pois são estudos que possibilitam ceder espaços para ouvir, observar e entender como se dá esse processo de cuidado na atenção primária. Portanto, conclui-se que os resultados desse escopo evidenciam a relevância da temática em novos estudos, aliados a práticas da integralidade do cuidado no pré-natal na atenção primária.

\section{Referências}

Ayres, J. R. C. M. (2001). Sujeito, intersubjetividade e práticas de saúde. Ciênc. saúde coletiva, 6(1): 63-72. 10.1590/S1413-81232001000100005.

Ayres, J. R. C. M. (2004). O cuidado, os modos de ser (do) ser humano. Saúde soc, 13(3): 16-29. 10.1590/S0104-12902004000300003.

Ayres, J. R. C. M. (2009). Organização das ações de atenção à saúde: modelos e práticas. Saúde soc, 18(Suppl 2): 11-23. 10.1590/S0104-12902009000600003.

Ayres, J. R. C. M., Carvalho, Y. M., Nasser, M. A., Saltão, R. M. \& Mendes, V. M. (2012). Ways of comprehensiveness: adolescents and young adults in Primary Healthcare. Interface, 16(40): 67-82. 10.1590/S1414-32832012005000021.

Barbiani, R., Nora, C. R. \& Schaefer, R. (2016). Nursing practices in the primary health care context: a scoping review. Rev Lat Am Enfermagem, 29 ,(24) :e2721. 10.1590/1518-8345.0880.2721

Barbieri, M. R. B. (2020). Mapeamento da integralidade do cuidado durante o pré-natal na atenção primária: scoping review. [dissertação]. São Carlos: Universidade Federal de São Carlos.

Barger, M., Faucher, M. A., Murphy, P. A. (2015). Part II: The Centering Pregnancy model of group prenatal care. J Midwifery Womens Health, 60(2): 211213. 10.1111/jmwh.12307

Baxley, S. M. \& Ibitayo, K. (2015). Expectations of pregnant women of Mexican origin regarding their health care providers. J Obstet Gynecol Neonatal Nurs, $15,44(3): 389-96.10 .1111 / 1552-6909.12572$

Bittencourt, S. D. A., Cunha, E. M., Domingues, R. M. S. M., Dias, B. A. S., Dias, M. A. B., Torres, J. A. \& Leal, M. C. (2020). Nascer no Brasil: continuity of care during pregnancy and postpartum period for women and newborns. Rev Saude Publica, 54:100. 10.11606/s1518-8787.2020054002021

Butler, M. M., Sheehy, L., Kington, M. M., Walsh, M. C., Brosnan, M. C., Murphy, M., Naughton, C., Drennan, J. \& Barry, T. (2015). Evaluating midwife-led antenatal care: choice, experience, effectiveness, and preparation for pregnancy. Midwifery, 31(4):418-425. 10.1016/j.midw.2014.12.002

Cardoso, T. Z. (2018). Avaliação do cuidado pré-natal nos serviços de Atenção Básica à Saúde do Piauí no contexto do Programa Nacional de Melhoria do Acesso e da Qualidade da Atenção Básica. Ribeirão Preto: Universidade de São Paulo.

Cecílio, L. C. O. \& Reis, A. A. C. (2018). Notes on persistent challenges for basic health care in Brazil. Cad Saúde Pública, 34(8), e00056917. 10.1590/0102$311 \times 00056917$.

Cunha, A. C., Lacerda, J. T., Alcauza, M. T. R. \& Natal, S. (2019). Evaluation of prenatal care in Primary Health Care in Brazil. Rev. Bras. Saúde Mater. Infant, 19(2):447-458. 10.1590/1806-93042019000200011. 
Delfino, M. R. R., Patrício, Z. M., Martins, A. S. \& Silvério, M. R. (2004). O processo de cuidar participante com um grupo de gestantes: repercussões na saúde integral individual-coletiva. Cienc. Saúde Colet, 9(4): 1057-1066. 10.1590/S1413-81232004000400026

Dias, M. S. A., Parente, J. R. F., Vasconcelos, M. I. O. \& Dias, F. A. C. (2014). Intersetorialidade e Estratégia Saúde da Família: tudo ou quase nada a ver? Cienc. Saúde Colet, 19(11): 4371-4382. 10.1590/1413-812320141911.11442014.

Domingues, R. M., Viellas, E. F., Dias, M. A., Torres, J. A., Theme-Filha, M. M., Gama S. G. \& Leal, M. C. (2015). Adequacy of prenatal care according to maternal characteristics in Brazil. Rev Panam Salud Publica, 37(3):140-7.

Duarte, S. J. H. (2012). Motivos que levam as gestantes a fazerem o pré-natal: um estudo das representações sociais. Ciencia y enfermeria, 18(2): 75-82. $10.4067 /$ S0717-95532012000200008

Genovesi, F. G., Canario, M. A. S. S., Godoy, C. B., Maciel, S. M., Cardelli, A. A. M. \& Ferrari, R. A. P. (2020). Maternal and child health care: adequacy indez in public health services. Rev. Bras. Enferm, 73(Suppl 4): e20170757. https://doi.org/10.1590/0034-7167-2017-0757

Goudard, M. J. F., Simões, V. M. F., Batista, R. F. L., Queiroz, R. C. S., Alves, M. T. S. S. B., Coimbra, L. C., Martins, M. G., Barbieri, M. A. \& Nathasje, I. F. (2016). Inadequação do conteúdo da assistência pré-natal e fatores associados em uma coorte no nordeste brasileiro. Cien Saúde Colet, 21(4):1227-1238. $10.1590 / 1413-81232015214.12512015$.

Guimarães, W. S. G., Parente, R. C. P., Guimarães, T. L. F. \& Garnelo, L. (2010). Acesso e qualidade da atenção pré-natal na Estratégia Saúde da Família: infraestrutura, cuidado e gestão. Cad Saúde Pública, 10 ,34(5):e00110417. 10.1590/0102-311X00110417

Hanson, L., Vandevusse, L., Roberts, J. \&Forristal, A. (2009). A critical appraisal of guidelines for antenatal care: components of care and priorities in prenatal education. J Midwifery Womens Health, 54(6): 458-468. 10.1016/j.jmwh.2009.08.002

Langer, A., Villar, J., Romero, M., Nigenda, G., Piaggio, G., Kuchaisit, C., Rojas, G., Al-Osimi, M., Belizán, J. M., Farnot, U., Al-Mazrou, Y., Carroli, G., Ba'aqueel, H., Lumbiganon, P., Pinol, A., Bergsjo, P., Bakketeig, L., Garcia, J. \& Berendes, H. (2002). Are women and providers satisfied with antenatal care? Views on a standard and a simplified, evidence-based model of care in four developing countries. BMC Womens Health. 2(1):7. 10.1186/1472-6874-2-7.

Lazarus, E. S. \& Philipson, E. H. (1990). A longitudinal study comparing the prenatal care of Puerto Rican and white women. Birth, 17(1): 6-11. 10.1111/j.1523-536x.1990.tb00002.x

Leal, M. C., Szwarcwald, C. L., Almeida, P. V. B., Aquino, E. M. L., Barreto, M. L., Barros, F. \& Victora, C . (2018). Saúde reprodutiva, materna, neonatal e infantil nos 30 anos do Sistema Único de Saúde (SUS). Ciência e Saúde Coletiva, 23(6): 1915-1928. 10.1590/1413-81232018236.03942018.

Livramento, D. V. P., Backes, M. T. S., Damiani, P. R., Castillo, L. D. R., Backes, D. S. \& Simão, A. M. S. (2019). Perceptions of pregnant women about prenatal care in primary health care. Rev Gaúcha Enferm, 40: e20180211. 10.1590/1983-1447.2019.20180211.

Marsciani, F. (2014). Subjetividade e intersubjetividade entre semiótica e fenomenologia. Galaxia, 14(28):10-19. 10.1590/1982-25542014221105.

Martinelli, K. G., Santos, N. E. T., Gama, S. G. N. \& Oliveira, A. E. (2014). Adequação do processo da assistência pré-natal segundo os critérios do Programa de Humanização do Pré-Natal e Nascimento e Rede Cegonha. Rev. Bras. Ginecol. Obstet, 36(2):56-64. 10.1590/S0100-72032014000200003.

Mathibe-Neke, J. M. (2008). The expectations of pregnant women regarding antenatal care. Curationis, 31(3): 4-11. 10.4102/curationis.v31i3.1008

Mattos, R. (2005) Os sentidos da integralidade: algumas reflexões acerca de valores que merecem ser defendidos. In: Pinheiro R. \& Mattos R, organizadores. Os Sentidos da Integralidade na Atenção e no Cuidado em Saúde. 4.ed. Rio de Janeiro: Cepesc/IMS/Uerj/Abrasco.

Ministério da Saúde. (1984). Assistência integral à saúde da mulher: bases de ação programática. Brasília: Centro de Documentação do Ministério da Saúde.

Ministério da Saúde. (2000). Portaria nº 569, de 1 de junho de 2000. Institui o Programa de Humanização do Pré-natal e Nascimento, no âmbito do Sistema Único de Saúde.

Ministério da Saúde. (2011). Portaria nº 1459, de 24 de junho de 2011. Institui, no âmbito do Sistema Único de Saúde, a Rede Cegonha.

McNeill, J. A. \& Reiger, K. M. (2015). Rethinking prenatal care within a social model of health: an exploratory study in Northern Ireland. Health Care Women Int, 36(1): 5-25. 10.1080/07399332.2014.900061

Nogueira, C. M. C. S., Sousa, C. N. S., Nóbrega, L. L. R., Sales, L. K. O. \& Morais, F. R. R. (2017). Prenatal care and practices developed by the health team: integrative review. Cuidado Fund. 9(1):279-288. 10.9789/2175-5361.2017.v9i1.279-288

Novick, G. (2009). Women's experience of prenatal care: an integrative review. J Midwifery Womens Health, 54(3): 227-237. 10.1016/j.jmwh.2009.02.003.

Parada, C. M. G. L. \& Tonete, V. L. P. (2008). O cuidado em saúde no ciclo gravídico puerperal sob a perspectiva de usuárias de serviços públicos. Interface, 12(24): 35-46. 10.1590/S1414-32832008000100004.

Peters, M. D. J., Godfrey, C. M., Khalil, H., McInerney, P., Parker, D. \& Soares, C. B. (2015). Guidance for conducting systematic scoping reviews. Int J Evid Based Healthc, 13(3): 141-146. 10.1097/XEB.0000000000000050.

Philippi, J. C., Myers, C. R. \& Schorn, M. N. (2014). Facilitators of prenatal care access in rural Appalachia. Women and Birth, 27(4): e28-35. 10.1016/j.wombi.2014.08.001.

Protasio, A. P. L., Silva, P. B., Lima, E. C., Gomes, L. B., Machado, L. S. \& Valença, A. M. G. (2014). Avaliação do sistema de referência e contrarreferência do estado de Paraíba segundo os profissionais da Atenção Básica no contexto do $1^{\circ}$ ciclo de Avaliação Externa do PMAQ-AB. Saúde Debate, 38(spe): 209220. https://doi.org/10.5935/0103-1104.2014S016. 
Research, Society and Development, v. 10, n. 12, e429101220639, 2021

(CC BY 4.0) | ISSN 2525-3409 | DOI: http://dx.doi.org/10.33448/rsd-v10i12.20639

Prudêncio, P. S. (2017). Avaliação da expectativa e satisfação da gestante com o cuidado pré-natal na Atenção Primária à Saúde. [tese]. Ribeirão Preto: Universidade de São Paulo.

Rios, C. T. F \& Vieira, N. F. C. (2007). Ações educativas no pré-natal: reflexão sobre a consulta de enfermagem como um espaço para educação em saúde. Cienc. Saúde Colet, 12(2): 477-486. 10.1590/S1413-81232007000200024.

Santos, A. L. M. \& Souza, M. H. T. (2017). Elaboration of new technologies in nursing: use a prevention booklet. Rev enferm UFPE, 11(10):3893-8. 10.5205/reuol.12834-30982-1-SM.1110201725

Santos, R. V. (2009). Integralidade do cuidado à gestante, puérpera e recém-nascido: o olhar de usuárias. [dissertação]. Belo Horizonte: Universidade Federal de Minas Gerais.

Silva, L. A. (2015). Assistência pré-natal na rede municipal de Niterói: a ótica valorativa dos profissionais de saúde. [dissertação]. Niterói: Universidade Federal Fluminense.

Silva, M. Z. N., Andrade, A. B. \& Bosi, M. L. M. (2014). Acesso e acolhimento no cuidado pré-natal à luz de experiências de gestantes na Atenção Básica. Saúde Debate, 38(103):805-816. 10.5935/0103-1104.20140073

Sodré, R. L. R. (2015). Atenção básica ao pré-natal e puerpério no estado de Goiás. [dissertação]. Goiânia: Universidade Federal de Goiás.

Tomasi, E., Fernandes, P. A. A., Fisher, T., Siqueira, F. C. V., Silveira, D. S., Thumé, E., \& Facchini, L. A. (2017). Quality of prenatal services in primary healthcare in Brazil: indicators and social inequalities. Cad de Saúde Pública, 33 (3), e00195815. 10.1590/0102-311x00195815.

Valdes, I. N. F., Santos, E. O. \& Prado, E. V. (2017). Programa mais médicos: qualificação da atenção ao pré-natal e puerpério no âmbito da estratégia de saúde da família. Rev. APS, 20(3): 403-413. https://doi.org/10.34019/1809-8363.2017.v20.16000

Zampieri, M. F. M. \& Erdmann, A. L. (2010). Cuidado humanizado no pré-natal: um olhar para além das divergências e convergências. Rev. Bras. Saúde Mater. Infant, 10(3): 359-367. 10.1590/S1519-38292010000300009

Warmling, C. M., Fajardo, A. P., Meyer, D. E. \& Bedos C. (2018). Práticas sociais de medicalização e humanização no cuidado de mulheres na gestação. Cad Saúde Pública, 29 ,34(4): e00009917. https://doi.org/10.1590/0102-311x00009917.

World Health Organization. (2016). Who recommendations on antenatal care for a positive pregnancy experience. https://www.who.int/reproductivehealth/publications/maternal_perinatal_health/anc-positive-pregnancy-experience/en/

Wright, D., Pincombe, J. \& Mckellar, L. (2018). Exploring routine hospital antenatal care consultations - An ethnographic study. Women and Birth, 31(3) :e162-e169. 10.1016/j.wombi.2017.09.010. 